\title{
Tratamiento del estado epiléptico refractario con topiramato oral
}

\author{
Bernardita Soler, Jaime G odoy, Patricio Mellado \\ Treatment of refractory \\ status epilepticus with topiramate. \\ Report of three cases
}

Refractory status epilepticus is a catastrophic illness of the central nervous system, with a mortality rate that reaches $50 \%$. We report three patients admitted with refractory status epilepticus: a 24 year-old male that discontinued antiepileptic medications, a 46 year-old male with a focal epilepsy secondary to an encephalitis that discontinued medications due to gastrointestinal problems and a 59 year-old male with an ischemic encephalopathy. All were treated with topiramate, delivered through a nasogastric tube with a good response (Rev Méd Chile 2009; 137: 936-9).

(Key words: Anticonvulsants; Status epilepticus; Topiramate)

Recibido el 14 de octubre, 2008. Aprobado el 10 de marzo, 2009.

Departamento de Neurología, Escuela de Medicina, Pontificia Universidad Católica de Chile. Santiago de Chile.

$\mathrm{N}_{\mathrm{s}}^{\circ}$ existe consenso sobre la definición de estado epiléptico refractario (EER), aceptándose, entre otras, aquel estado epiléptico (EE) que no responde a dos o tres anticonvulsivantes de primera línea ${ }^{1}$. Es una condición catastrófica del sistema nervioso central, llegando su mortalidad hasta $50 \%^{2}$. Su tratamiento tampoco ha sido definido y se basa principalmente en recomendaciones de expertos fundadas en pocas series, pequeñas y no controladas ${ }^{3}$.

Se han publicado algunas experiencias de pacientes con EER tratados exitosamente con topiramato (TPM) oral, un anticonvulsivante de última generación ${ }^{4-6}$. Presentamos nuestra experiencia con tres pacientes consecutivos con EER que respondieron precozmente y sin complicaciones a TPM oral.

Correspondencia a: Dr. Patricio Mellado Talesnik. Marcoleta 352. Santiago Chile. Teléfono: 3543316.

E mail: pmellado@med.puc.cl

\section{CASOS CĹNICOS}

Paciente 1. Hombre de 24 años, antecedentes de epilepsia focal, con crisis parciales complejas con y sin generalización secundaria desde los dos años. Bajo tratamiento con lamotrigina y carbamazepina, con niveles séricos en rangos terapéuticos óptimos (RTO), la frecuencia de crisis era de una cada 2 ó 3 meses. Influenciado por una tercera persona suspendió los fármacos, desarmollando días después episodios de confusión, los que fueron aumentando en frecuencia y duración. Diez días después se pesquisó en un electroencefalograma (EEG) un EE no convulsivo. Ingresó a la unidad de cuidados intensivos, donde recibió varios esquemas terapéuticos, incluyendo coma barbitúrico, sin obtener respuesta favorable. Con esta última terapia se logró el control transitorio del EER, el que reapareció con caracteres semejantes a los iniciales al tercer día de suspendido este tratamiento.

Siete días después se agregó TPM por sonda nasoenteral (SNE) en dosis de $200 \mathrm{mg} /$ día, con incrementos progresivos hasta alcanzar al tercer día $800 \mathrm{mg} /$ día, momento en que se consiguió el control del EER, con desaparición de las crisis electroclíni- 
cas. Se mantuvo hospitalizado por 46 días por presentar crisis epilépticas esporádicas, controladas por completo a los 27 días de evolución.

A los 2 meses retomó su vida laboral, en terapia con TPM $100 \mathrm{mg} /$ día, presentando una crisis tónico clónico generalizada al mes (Tablas 1 y 2).

Paciente 2. Hombre de 46 años, presentó a los 21 años una encefalitis idiopática, quedando secuelado con demencia y epilepsia focal, con crisis parciales complejas y secundariamente generalizadas, en promedio 2 a 4 al mes, bajo terapia con valproato y barbitúricos; tenía antecedentes de alergia a la fenitoína.

La semana previa a su ingreso inició dolor abdominal y vómitos, requiriendo la suspensión de ingesta por vía enteral, dejando de recibir terapia anticonvulsivante. Ingresó a nuestro hospital con un síndrome confusional, cuyo estudio EEG demostró un $\mathrm{EE}$. Se reinició tratamiento farmacológico con valproato intravenoso $(20 \mathrm{mg} / \mathrm{kg})$, primidona, fenobarbital y clobazam. Por persistencia de las crisis se agregó infusión continua de midazolam intravenoso $0,5 \mathrm{mg} /$ $\mathrm{kg}$. Se inició EEG continuo que mostró persistencia del EER. Al tercer día se agregó TPM por SNE en dosis de 200 mg/día. Dos días después se controló el EER, volviendo a presentar crisis parciales complejas aisladas (1/semana). Fue dado de alta a los 15 días con su terapia de base (Tablas 1 y 2).

Paciente 3. Hombre de 59 años, antecedentes de obesidad mórbida y tabaquismo, presentó un paro

Tabla 1. Antecedentes de los pacientes en estado epiléptico refractario tratados con topiramato oral

\begin{tabular}{|ccclccc|}
\hline Paciente & $\begin{array}{c}\text { Edad } \\
\text { (años) }\end{array}$ & Sexo & Antecedentes & $\begin{array}{c}\text { Etiología } \\
\text { de la epilepsia } \\
\text { de crisis }\end{array}$ & $\begin{array}{c}\text { Frecuencia } \\
\text { previa }\end{array}$ & $\begin{array}{c}\text { Factor } \\
\text { descompensante }\end{array}$ \\
\hline 1 & 24 & Masculino & $\begin{array}{l}\text { Epilepsia focal } \\
\text { desde los } 2 \text { años } \\
\text { Demencia secundaria, } \\
\text { epilepsia parcial, } \\
\text { alergia fenitoína } \\
\text { Obesidad. } \\
\text { Tabaquismo crónico }\end{array}$ & $\begin{array}{l}\text { Criptogénica } \\
\text { Encefalitis idiopática }\end{array}$ & $\begin{array}{l}\text { Encélopatía } \\
\text { hipóxico isquémica }\end{array}$ & $\begin{array}{c}\text { Suspensión } \\
\text { fármacos } \\
\text { Suspensión } \\
\text { fármacos }\end{array}$ \\
\hline & 59 & Masculino & \\
\hline
\end{tabular}

Tabla 2. D escripción de los pacientes en estado epiléptico refractario tratados con topiramato oral

\begin{tabular}{|c|c|c|c|c|c|}
\hline Paciente & $\begin{array}{l}\text { Anticonvulsivantes } \\
\text { antes del EE }\end{array}$ & $\begin{array}{c}\text { Terapia del EER } \\
\text { previo TPM }\end{array}$ & $\begin{array}{l}\text { Duración EER } \\
\text { antes de TPM }\end{array}$ & $\begin{array}{l}\text { Tiempo TPM } \\
\text { hasta control EER }\end{array}$ & $\begin{array}{c}\text { D osis TPM } \\
\text { que controla EER }\end{array}$ \\
\hline 1 & $\begin{array}{l}\text { Carbamazepina } \\
\text { Lamotrigina }\end{array}$ & $\begin{array}{l}\text { Carbamazepina } \\
\text { Lamotrigina } \\
\text { Fenitoína } \\
\text { Valproato } \\
\text { Levetiracetam } \\
\text { Midazolam } \\
\text { Fenobarbital } \\
\text { Tiopental }\end{array}$ & 17 días & $72 \mathrm{~h}$ & $400 \mathrm{mgc} / 12 \mathrm{~h}$ \\
\hline 2 & $\begin{array}{l}\text { Valproato } \\
\text { Fenobarbital } \\
\text { Primidona }\end{array}$ & $\begin{array}{l}\text { Clobazam } \\
\text { Valproato } \\
\text { Primidona } \\
\text { Midazolam }\end{array}$ & 3 días & $48 \mathrm{~h}$ & $100 \mathrm{mgc} / 12 \mathrm{~h}$ \\
\hline 3 & & $\begin{array}{l}\text { Fenitoína } \\
\text { Valproato } \\
\text { Primidona } \\
\text { Midazolam }\end{array}$ & 14 días & $48 \mathrm{~h}$ & $200 \mathrm{mg} \mathrm{c} / 12 \mathrm{~h}$ \\
\hline
\end{tabular}

EER: estado epiléptico refractario, TPM: topiramato 
cardiomespiratorio 15 días antes de su ingreso, los diagnósticos fueron: encefalopatía hipóxico-isquémica (EHI) y EER no convulsivo. El cuadro epiléptico se había intentado controlar sin éxito con valproato, fenitoína y clonazepam, todos en RTO.

En nuestro hospital se inició EEG continuo que evidenció persistencia de las crisis, se agregó TPM por SNE en dosis de $200 \mathrm{mg} /$ día. Al segundo día se aumentó a $400 \mathrm{mg} /$ día, lográndose controlar el EER. El paciente despertó a los 15 días en estado de mínima conciencia, fue dado de alta a los 40 días, sin crisis convulsivas (Tablas 1 y 2 ).

\section{Discusión}

Clásicamente se ha definido EE como aquella crisis epiléptica que se prolonga por más de $30 \mathrm{~min}$, o a la repetición de las mismas sin recuperación completa de conciencia ${ }^{2,7}$. Sin embargo, dado la gravedad del cuadro, a que en promedio una crisis convulsiva dura $1 \mathrm{~min}$ y a que la extensa mayoría se detiene espontáneamente antes de los $4 \mathrm{~min}$, se ha propuesto una nueva definición temporal para $\mathrm{EE}$, quedando este en $5 \mathrm{~min}^{1}$. La refractariedad del $\mathrm{EE}$ no se encuentra definida, sugiriéndose como criterios la falta de respuesta a dos o tres drogas de primera línea o la prolongación por más de $60 \mathrm{~min}^{4,8,9}$ hasta $2 \mathrm{~h}^{10}$.

Datos retrospectivos estiman que la prevalencia del EER corresponde a 1/3 de los $\mathrm{EE}^{9}$. Su pronóstico depende fundamentalmente de su etiología ${ }^{1}$, resultando relevante también su duración. Así, a mayor tiempo en EE, mayor dificultad para lograr su remisión 11 , lo que se explica por cambios estructurales en la membrana citoplasmática neuronal, constatándose en modelos experimentales una reducción del número de receptores GABA A e incremento de receptores glutamatérgicos (NMDA). Se ha evaluado la eficacia de diferentes fármacos anticonvulsivantes en el EE, estimándose que la combinación de lorazepam y fenitoína en dosis máxima y por vía intravenosa es el tratamiento más útil. Sin embargo, no existen estudios metodológicamente adecuados que evalúen la utilización de otros anticonvulsivantes ${ }^{2,12}$.

El tratamiento farmacológico del EER se basa sólo en experiencias personales y limitadas. Los fármacos más utilizados son: coma benzodiazepíni$\mathrm{co}^{3,13}$ y anestésicos ${ }^{14,15}$. Un estudio presentó un grupo de pacientes con EER que respondieron sólo al uso de TPM ${ }^{4-6}$. El TPM es un anticonvulsivante eficaz y con baja incidencia de efectos adversos ${ }^{16}$. Su cinética es lineal, con una vida media de $21 \mathrm{~h}$, baja unión a proteínas, sin autoinducción enzimática y no produce metabolitos activos ${ }^{17}$. Se elimina por vía renal, principalmente como fármaco no modificado ${ }^{17}$. Presenta varios mecanismos de acción: bloqueo de los canales de sodio y calcio, acción gabaérgica, antagonismo glutamatérgico e inhibición de la enzima anhidrasa carbónica ${ }^{18,19}$. Sus efectos adversos son infrecuentes, destacando la disminución de las capacidades cognitivas y el glaucoma agudo de ángulo estrecho idiosincrático ${ }^{20}$.

Towne et al presentan una serie de 6 pacientes adultos en EER que respondieron a TPM oral en dosis que fluctuaron entre 300 a 1.600 mg/día entre 6 h y 10 días después de iniciado el fármaco: el paciente que demoró más en responder fue el que llevaba más tiempo en EER (38 días) y en él se utilizó la dosis más alta (1.600 mg/día). La única complicación fue somnolencia ${ }^{4}$. Bensalem et al describieron tres pacientes con EER que respondieron a TPM en dos días: la dosis fueron de $1 \mathrm{~g} /$ día por 2 a 5 días, las que fueron disminuidas gradualmente en los días subsiguientes sin que los pacientes presentaran complicaciones ${ }^{5}$. Esta terapia ha sido utilizada también en niños ${ }^{6}$ con resultados semejantes. Nuestros tres pacientes con EER fueron documentados con EEGs, dos tenían antecedentes de epilepsia de años de evolución, estables en el número de crisis bajo terapia farmacológica y en ellos la causa del EER fue la suspensión del tratamiento, uno de los más frecuentes desencadenantes de EE en pacientes con historia conocida de epilepsia; en el tercer caso fue desencadenado por una EHI. Todos persistieron en EE a pesar del tratamiento con anticonvulsivantes (Tabla 2). Debido a la resistencia a tratamiento se decidió agregar TPM en dosis de $200 \mathrm{mg} /$ día por vía oral, la que debió aumentarse en dos casos, en uno de ellos hasta 400 $\mathrm{mg} /$ día. Puesto que no existe un esquema de tratamiento con TPM para el EER, la dosis inicial utilizada fue la propuesta por Towne et $\mathrm{al}^{4}$. Bensalem et al utilizaron una dosis inicial mayor ( $1 \mathrm{~g} /$ día), sin que se presentaran complicaciones. En nuestros pacientes la respuesta fue rápida, controlándose el EER en todos los casos dentro de las primeras $72 \mathrm{~h}$ de uso de TPM, sin que observásemos efectos adversos adjudicables a esta terapia. Dos pacientes fueron dados de alta en sus condiciones basales, mientras que el paciente que sufrió una EHI permaneció en 
estado de mínima conciencia, logrando después de un año comprender órdenes simples.

Muchos de los fámacos utilizados en el EER producen complicaciones sistémicas, en particular depresión respiratoria y compromiso hemodinámico, por lo que frecuentemente estos pacientes deben ser conectados a un ventilador mecánico y apoyados con drogas vasoactivas que deben ser administradas por vía central ${ }^{2,19}$. El TPM no presenta estas complicaciones, lo que constituye una ventaja. La carencia de niveles plasmáticos que permitan optimizar la dosis (especialmente en pacientes críticos, que habitualmente reciben múltiples fármacos) y la falta de presentaciones parenterales son en cambio, dos importantes limitaciones. Es posible que la multiplicidad de mecanismos de acción propuestos para esta droga explique su eficacia en el EER, condición en la

\section{REFERENCIAS}

1. LowESTEIN DH. The management of refractory status epilepticus: an update. Epilepsia 2006; 47: 35-40.

2. Melado P, Godoy J. Estado epiléptico en el adulto. En: Castillo L, Romero C, Mellado P. (eds) Cuidados Intensivos Neurológicos. Santiago. Mediterráneo. 2004; 408-23.

3. Claassen J, Hirsch LJ, Emerson RG, Mayer SA. Treatment of refractory status epilepticus with pentobarbital, propofol, or midazolam: A systematic review. Epilepsia 2002; 43: 146-53.

4. Towne AR, Garnett LK, Watterhouse EJ, Morton LD, Delorenzo RJ. The use of topiramate in refractory status epilepticus. Neurology 2003; 60: 332-4.

5. Bensalem MK, Fakhoury TA. Topiramate and status epilepticus: report of three cases. Epilepsy and Behavior 2003; 4: 757-60.

6. Kahriman M, Minecan D, Kutluay E, Selwal L, Beydoun A. Efficace of topiramate with refractory status epilepticus. Epilepsia 2003; 44: 1353-6.

7. De Giorgio CM, Treiman DM. Status epilepticus. In: Noseworthy JH. (ed). Neurological Therapeutics. Principles and Practice. London. Martin Dunitz. 2003; 316-29.

8. GatTANIS JN, DrisLane FW. Status epilepticus: a review of different syndromes, theirs current evaluation, and treatment. The Neurologist 2003; 9: 61-76.

9. Mayer SA, Claassen J, Lokin J, Mendeisohn F, Dennis LJ, Fitzsimmons BF. Refractary status epilepticus, frecuency, risk factors and outcome. Arch Neurol 2003; 59: 205-10.

10. Prasad A, Worrall BB, Bertrán EH, Bieck TP. Propofol and midazolam in the treatment of refractory status epilepticus. Epilepsia 2001; 42: 380-6. que es esperable la resistencia a los fármacos habitualmente usados, especialmente los gabaérgicos, dada la existencia de cambios estructurales a nivel de los receptores neuronales.

\section{CONCLUSIÓN}

El EER es una enfermedad catastrófica del SNC con una mortalidad de hasta $50 \%$, para la que no existen esquemas terapéuticos establecidos. Es necesario incrementar las opciones terapéuticas, especialmente para los casos más resistentes. El uso de TPM oral puede ser una de ellas, como ha sido reportado en la literatura y mostrado en nuestros casos. La validación de esta terapia requiere de estudios controlados y con mayor número de pacientes.

11. Payne TA, Bieck TP. Status epilepticus. Crit Care Clin 1997; 13: 17-38.

12. WijDICKS EFM. Status epilepticus. In: Wijdicks EFM (ed). The clinical practice of critical care neurology. Oxford. Oxford University Press. 2003; 389-402.

13. Claassen J, Hirsch LJ, Emerson RG, Bates JE, Thompson TB, MAYER SA. Continuous EEG monitoring and midazolam infusion for refractory nonconvulsive status epilepticus. Neurology 2001; 57: 1036-42.

14. Sharpe MD, Young GB, Mirsattari S, Harris CH. Prolonged desflurane administration for refractory status epilepticus. Anesthesiology 2002; 97: 261-4.

15. Parviainen A, Usaro A, Kälviäinen R, Kauzanen E, Mervadia E, Ruokonen E. High-dose thiopental in the treatment of refractory status epilepticus in intensive care unit. Neurology 2002; 59: 1249-51.

16. Privitera MD, Twyman RE. Topiramate: Clinical efficacy and use in epilepsy. En: Levy R, Mattson R, Meldrum B, Perucca E. (ed). Antiepileptic Drugs. Philadelphia. Lippincott Williams and Wilkins. 2002; 740-52.

17. DOOSE DR, STREETER AJ. Topiramate: Chemistry, biotransformation and pharmacokinetics. En: Levy R, Mattson R, Meldnum B, Perucca E. (ed). Antiepileptic Drugs. Philadelphia. Lippincott Williams and Wilkins. 2002; 727-34.

18. WHITE HS. Topiramate: Mechanism of action. En: Levy R, Mattson R, Meldrum B, Perucca E. (ed). Antiepileptic Drugs. Philadelphia. Lippincott Williams and Wilkins. 2002; 719-26.

19. AвEND N, Dlugos D. Treatment of refractory status epilepticus. Literature review and a proposed protocol. Pediatr Neurol 2008; 38: 377-90.

20. Sachdeo RC, Karia RM. Topiramate: Adverse effects. En: Levy R, Mattson R, Meldrum B, Perucca E. (ed). Antiepileptic Drugs. Philadelphia. Lippincott Williams and Wilkins. 2002; 760-4. 